\title{
Adaptación de cofias metálicas de Co-Cr realizadas sobre dos líneas de terminación y fabricadas con dos técnicas
}

Adaptation of $\mathrm{Co}-\mathrm{Cr}$ metal copings made on two termination lines and manufactured with two techniques.

Enrique Manuel Gutiérrez Chanjónn ${ }^{1, a ; 2, b}$, Diana Esmeralda Castillo Andamayo ${ }^{2, a, b, c, ~ M a r t i ́ n ~ G i l b e r t o ~}$ Quintana del Solar ${ }^{2, a, b, d}$

\section{RESUMEN}

Las nuevas tecnologías digitalizadas han permitido migrar de la técnica de cera perdida colada por centrifugación (CPC) a la fabricación sustractiva por CAD/CAM como la técnica de fresado de bloque blando (SMB). Objetivo: El propósito de este estudio fue comparar las adaptaciones marginales e internas en cofias metálicas de Co- $\mathrm{Cr}$ sobre dos líneas de terminación: chamfer y bisel confeccionadas mediante dos técnicas: CPC y SMB. Material y Métodos: Se confeccionaron dos modelos maestros de Co-Cr con terminación chamfer y bisel, se formaron 4 grupos de estudio y se fabricaron 13 cofias por grupo. Para la evaluación de las adaptaciones marginal e interna se usó el método de la réplica en silicona, luego con un estéreo microscopio se evaluó las discrepancias marginales e internas. Resultados: Para la discrepancia marginal entre las dos técnicas, para la terminación bisel sólo se observó diferencias estadísticamente significativas $(\mathrm{p}<0,05)$ en los puntos A1 y A2 y en la terminación chamfer se observó diferencias estadísticamente significativas $(\mathrm{p}<0,05)$ en los puntos A1 y F1. En la adaptación interna comparando ambas técnicas en la terminación bisel se obtuvo diferencias estadísticamente significativas $(p<0,05)$ en los puntos E1, E2, D1, C2 y D2. En la terminación chamfer los resultados mostraron diferencias estadísticamente significativas $(\mathrm{p}<0,05)$ en todos los puntos. Conclusiones: Las cofias que presentaron mejor adaptación marginal fueron las confeccionadas por SMB con la línea de terminación chamfer, en la línea de terminación bisel no se pudo concluir cuál de las dos técnicas es mejor en adaptación marginal. Las que presentaron mejor adaptación interna en ambas líneas de terminación, en la zona axial fueron las cofias realizadas mediante la técnica CPC y en la zona oclusal presentaron mejor adaptación interna el SMB.

PALABRAS CLAVE: CAD/CAM, preparación del diente, adaptación marginal del diente.

Facultad de Odontología, Universidad Nacional Federico Villarreal. Lima, Perú.

Facultad de Estomatología, Universidad Peruana Cayetano Heredia. Lima, Perú.

Cirujano Dentista.

Especialista en Rehabilitación Oral

Magister en Estomatología. Docente del Departamento Académico de Odontología Social.

Magister en Estomatología. Docente del Departamento Académico de Clínica Estomatológica. 


\section{SUMMARY}

The new digitized technologies have allowed to migrate from the technique of lost cast wax by centrifugation (CPC) to the subtractive manufacturing by CAD / CAM as the technique of soft-block milling (SMB). Objective: The purpose of this study was to compare the marginal and internal adaptations in metal Co-Cr copes on two termination lines: chamfer and bevel made by two techniques: CPC and SMB. Material and Methods: Two Co$\mathrm{Cr}$ master models with chamfer and bevel termination were made, 4 study groups were formed and 13 copings were made per group. For the evaluation of the marginal and internal adaptations the replication method was used in silicone, then with a stereomicroscope the marginal and internal discrepancies were evaluated. Results: For the marginal discrepancy between the two techniques, only statistically significant differences were observed for the bevel termination $(\mathrm{p}<0.05)$ in points $\mathrm{A} 1$ and $\mathrm{A} 2$ and in the chamfer termination statistically significant differences were observed $(\mathrm{p}<0.05)$ in points $\mathrm{A} 1$ and $\mathrm{F} 1$. In the internal adaptation comparing both techniques in the bevel termination, statistically significant differences were obtained $(p<0.05)$ in points E1, E2, D1, C2 y D2. And in the chamfer termination, the results showed statistically significant differences $(p<0.05)$ in all the points. Conclusions: The copes that presented better marginal adaptation were those made by SMB with the chamfer termination line, in the bevel termination line it was not possible to conclude which of the two techniques is better in marginal adaptation. The ones that presented better internal adaptation in both lines of termination, in the axial zone were the copings made using the CPC technique and in the occlusal area the SMB had better internal adaptation.

\section{KEYWORDS: CAD/CAM , tooth preparation, dental marginal adaptation.}

\section{INTRODUCCIÓN}

La adaptación marginal e interna de las coronas son un punto muy importante para la rehabilitación protésica, una inadecuada adaptación marginal puede producir irritación gingival, disolución del cemento y subsecuente fracaso del tratamiento. A su vez también tienen influencia el diseño de la preparación, los procedimientos de laboratorio y la cementación. La línea de terminación es la configuración del margen cervical de una preparación dentaria, podemos usar: chamfer, hombro bisel y bisel de $135^{\circ}(1-8)$. Muchos investigadores vienen reportando cuál de los diseños de la línea de terminación presenta menor discrepancia marginal y una buena adaptación, pero aún existe controversia y actualmente no hay un consenso de cuál de estas líneas de terminación es la más adecuado para la confección de coronas. En restauraciones metalcerámicas algunos autores sugieren que la línea de terminación más adecuada es el tipo chamfer, porque permite tener buena adaptación marginal además de dar mayor espacio a nivel cervical para el opáquer y la cerámica feldespática. Otros autores recomiendan el tipo bisel en $135^{\circ}$, permitiendo tener buena adaptación marginal pero no tiene mucho espacio a nivel cervical para el material de revestimiento (9-16).
La adaptación marginal es el adecuado ajuste que debe existir entre la línea de terminación y el margen cervical de la restauración, la distancia perpendicular que existe entre estos dos puntos se conoce como discrepancia marginal, cuando hay una menor discrepancia marginal existe una mejor adaptación marginal. Una buena adaptación marginal disminuye la probabilidad de caries, enfermedad periodontal y fracaso de la restauración (17). La adaptación interna es el ajuste que se da entre la estructura de la restauración y la superficie del pilar dentario, la distancia perpendicular que existe entre estos dos puntos se conoce como discrepancia interna, esta distancia debe ser uniforme para proporcionar un espacio adecuado al agente cementante, proporcionar retención y resistencia adecuada (18). La mayoría de autores coinciden que la discrepancia marginal no debe ser mayor a $120 \mu \mathrm{m}$, en la discrepancia interna no hay aún un consenso entre los autores, pero la gran mayoría coincide en que debe ser uniforme, pudiendo oscilar en un rango de 50 a $350 \mu \mathrm{m}$ (17-20).

La aleación Co-Cr se puede utilizar con los sistemas convencionales de colado o mediante sistemas digitalizados CAD/CAM para fresado y láser (21-28). Para la obtención de cofias metálicas se puede utilizar la técnica CPC, que consiste en encerar un patrón de 
cera, posteriormente la cera es evaporada por calor en un horno, mediante un soplete se funde el metal y por último se impulsa el metal fundido por medio de una fuerza centrífuga en el molde a través de un canal denominado bebedero (29-32). Dentro de los sistemas $\mathrm{CAD} / \mathrm{CAM}$ existe la técnica aditiva y la sustractiva, en la técnica sustractiva se puede utilizar el fresado de bloques duros sinterizados o se puede utilizar la soft milling block o fresado de bloque blando (SMB) que utiliza bloques presinterizados. Para la elaboración de una restauración por este método se deben seguir tres fases: digitalización de la preparación dental obtenida por medio de un escáner, diseño de la restauración mediante un programa de computador y fresado del bloque presinterizado para obtener la restauración, luego se completa la sinterización en un horno con gas Argón (33-35).

Kim et al. evaluaron la adaptación marginal de cofias Co-Cr con tres sistemas: sustractivo en SMB de Ceramill ${ }^{\circledR}$ Sintron (Amann Girrbach AG, Koblach, Alemania), método aditivo en sinterizado selectivo por láser-SLS, y el método CPC. Se llegó a la conclusión que las prótesis fijas confeccionadas mediante el SMB presentaban una adaptación marginal menor $(32,6 \pm 4,8 \mu \mathrm{m})$ seguida por la SLS $(47,3 \pm 8,6 \mu \mathrm{m})$ y la CPC $(64,1 \pm 14,2 \mu \mathrm{m})$ llegando a la conclusión que el grupo de las cofias SMB tenían mejor adaptación marginal en comparación con la CPC (15).

Este estudio aporta evidencia científica y nuevos conocimientos en cuanto a niveles de adaptación marginal e interna en cofias metálicas unitarias en Co-Cr, con diferentes líneas de terminación chamfer y bisel generadas mediante las técnicas CPC y SMB. En nuestra realidad peruana la técnica de $\mathrm{CPC}$ es la más usada para la fabricación de restauraciones protésicas por los técnicos dentales, esta comparación con la técnica SMB proporcionaría una mayor información científica, teniendo relevancia clínica porque brindara información acerca de cuál de las técnicas nos permite lograr la mejor adaptación marginal e interna, evitando así las posibles complicaciones biológicas, técnicas y estéticas de las restauraciones con prótesis fijas, y así poder dar al paciente un tratamiento con mejor calidad.

El propósito de este estudio fue comparar in vitro la adaptación marginal e interna de cofias metálicas unitarias de aleación Co-Cr fabricadas con las técnicas cera perdida colada por centrifugación (CPC) y técnica de fresado de bloque blando (SMB) en $C A D /$ $C A M$, realizadas sobre dos líneas de terminación: chamfer y bisel.

\section{MATERIAL Y MÉTODOS}

Sehizo una preparación dentaria sobre una pieza natural premolar superior realizando una línea de terminación chamfer y bisel con un ángulo de convergencia de $12^{\circ}$, luego se duplicó la preparación en CAD/CAM creando dos modelos maestros de $\mathrm{Co}-\mathrm{Cr}$ (figura 1 y figura 2). Se tomaron impresiones con silicona de adición fluida y pesada, formando grupos por cada línea de terminación, cada grupo de 13 modelos de yeso tipo IV para la técnica de CPC utilizando pastillas de aleación Co- Cr CROMORON Premium C (DG DENTAL d.o.o, Gornji Grad, Slovenia), también se formó grupos por cada línea de terminación, cada grupo de 2 modelos de yeso para la SMB en CAD/ CAM se trabajó con el sistema Ceramill@ (Amann Girrbach AG, Koblach, Alemania) utilizando bloques blandos pre sinterizados de Co-Cr Ceramill ${ }^{\circledR}$ Sintron (Amann Girrbach AG, Koblach, Alemania). En los modelos de yeso de ambas líneas de terminación para la técnica de CPC de acuerdo al procedimiento de confección de cofias se aplicó un sellador de poros o

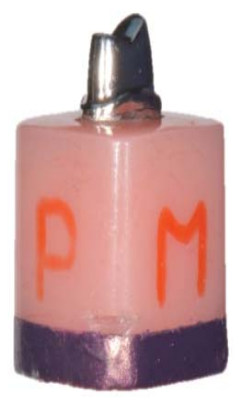

Figura 1. Modelo maestro en Co-Cr, chamfer

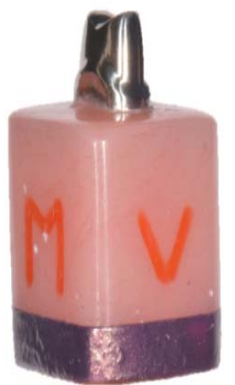

Figura2. Modelo maestro en $\mathrm{Co}-\mathrm{Cr}$, bisel. 
endurecedor de $5 \mu \mathrm{m}$ llamado die:master dúo (Renfert $\mathrm{GmbH}$, Hilzingen, Germany) en toda la preparación, luego se aplicó dos capas del espaciador Pico-Fit (Renfert GmbH, Hilzingen, Germany) de $20 \mu \mathrm{m}$ a 1 milímetro por encima de la línea de terminación y finalmente una capa de aislante para cera Picosep (Renfert $\mathrm{GmbH}$, Hilzingen, Germany) de $5 \mu \mathrm{m}$ en toda la preparación, obteniendo las medidas para el espacio del agente cementante de $10 \mu \mathrm{m}$ en la zona cervical y de $50 \mu \mathrm{m}$ en la zona interna. Para la técnica de SMB en CAD/CAM de acuerdo al procedimiento de confección de cofias, se escaneo los modelos de yeso con el Ceramill ${ }^{\circledR}$ map400 (Amann Girrbach AG, Koblach, Alemania) y se diseñó las cofias con el software Ceramill ${ }^{\circledR}$ Mind (Amann Girrbach AG, Koblach, Alemania), luego se freso con el Ceramill ${ }^{\circledR}$ Motion 2 (Amann Girrbach AG, Koblach, Alemania) los bloques blandos pre sinterizados de $\mathrm{Co}-\mathrm{Cr}$ Ceramill® Sintron (Amann Girrbach AG, Koblach, Alemania). Una vez terminado el fresado, se retiró los bloques para realizar el recorte unitario de las cofias,

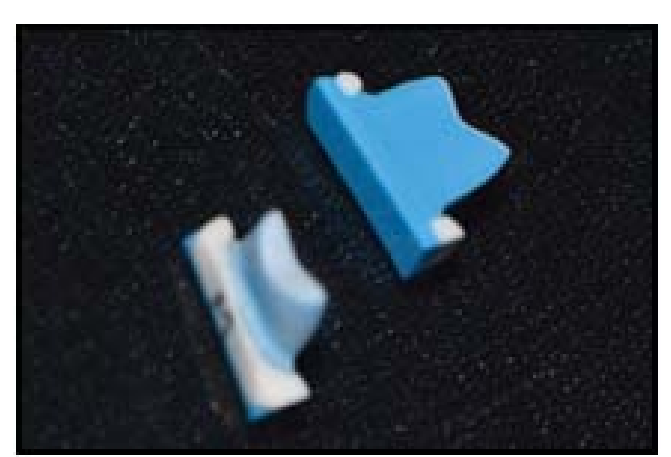

Figura 3: Replica de silicona Fit Checker ${ }^{\mathrm{TM}}$ Advance luego se culminó el proceso de sinterizado con el horno Ceramill@ Argotherm2 (Amann Girrbach AG, Koblach, Alemania) con gas argón. Para las cofias de la técnica de SMB se realizó una prueba piloto con la finalidad de determinar las medidas adecuadas para el espacio del agente cementante, donde se evaluó el asentamiento y sellado marginal de la cofia en el modelo maestro a través de la inspección visual, el uso de la punta de un explorador y con la técnica de la réplica de silicona, se determinó las medidas de $25 \mu \mathrm{m}$ en la zona cervical y de $50 \mu \mathrm{m}$ en la zona interna para ambas líneas de terminación. Las cofias de $\mathrm{Co}-\mathrm{Cr}$ fueron agrupadas en 13 cofias por grupo, haciendo un total de 52 cofias, la adaptación marginal e interna fue evaluada con la técnica de réplica de silicona VPES Fit Checker ${ }^{\mathrm{TM}}$ Advance (GC Corporation, Tokyo, Japan), esta se inyectó dentro de las cofias y se asentó sobre el modelo maestro a una fuerza de $15 \mathrm{~N}$ durante dos minutos con una máquina de ensayo universal Instron CTM-5L (LG Electronics, Seúl, Korea). Se retiró la cofia del modelo maestro y se inyectó sobre la VPES silicona fluida de adición obteniendo la réplica de silicona, se realizaron dos cortes en sentido vestíbulo-palatino y mesio-distal (figura 3). La numeración " 1 " correspondió al corte en sentido vestíbulo-palatino donde el punto A1 fue el punto marginal vestibular y F1 fue el punto marginal palatino, B1 y E1 representaron las zonas axiales, C1 y D1 la zona oclusal. La numeración "2" correspondió al corte en sentido mesio-distal donde el punto A2 fue el punto marginal mesial y F2 fue el punto marginal distal, B2 y E2 representaron las zonas axiales, $\mathrm{C} 2$ y D2 las zonas oclusales (figura 4

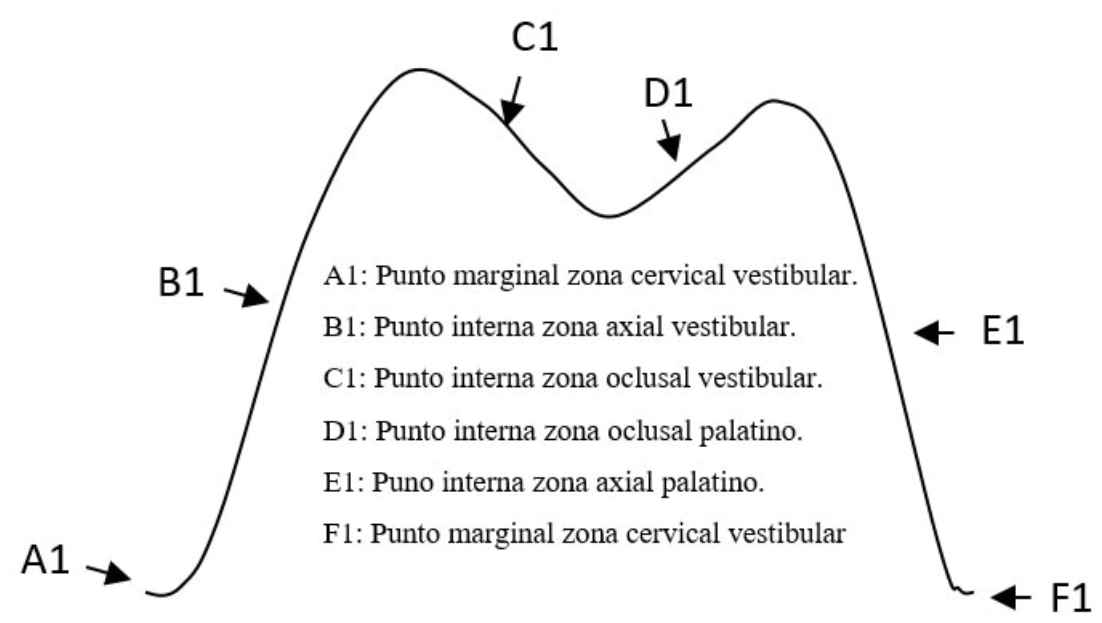

Figura 4. Corte sentido vestíbulo-palatino 


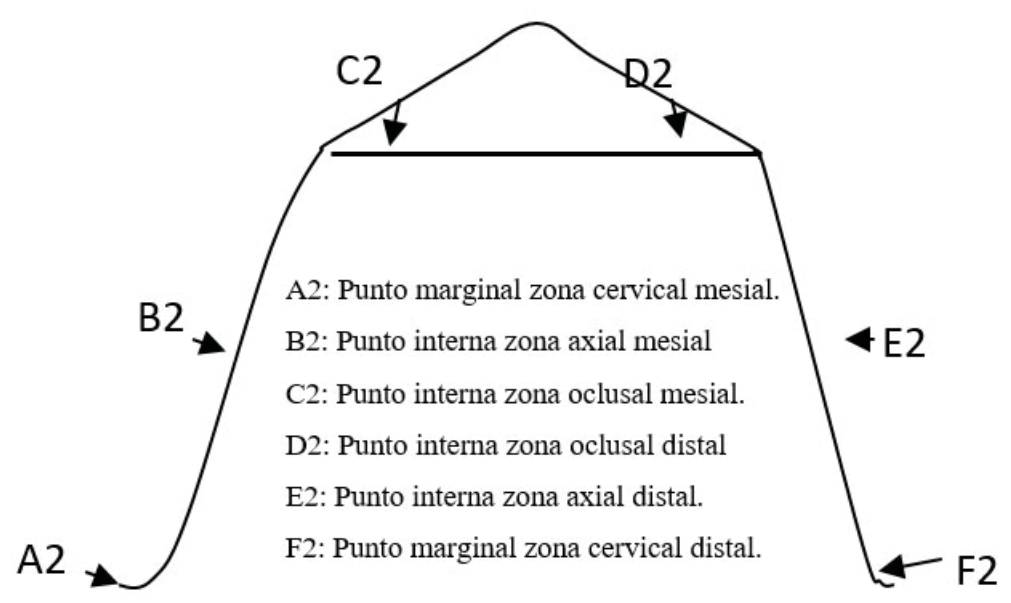

Figura 5. Corte sentido mesio-distal

y figura 5). Se evaluó las discrepancias marginales e internas en un estéreomicroscopio Greenough Leica S8 APO (Leica Biosystems GmbH, Wetzlar, Germany) a un aumento de 40X con el software Leica Application Suite LAS v3.4 (Leica Biosystems GmbH, Wetzlar, Germany) medidos en micrómetros $(\mu \mathrm{m})$. Se utilizó el programa STATA versión 12 para el análisis univariado y bivariado, donde se evaluaron los supuestos de normalidad con la prueba de Shapiro-Wilk. Se empleó la prueba de t de Student para distribución normal y la prueba de U MannWhitney si no se presentó distribución normal. El presente estudio utilizó un nivel de confianza al 90\% $(\mathrm{p}<0,05)$.

\section{RESULTADOS}

En la tabla 1 se muestran los promedios de la discrepancia marginal en cofias metálicas unitarias de aleación $\mathrm{Co}-\mathrm{Cr}$ realizadas por la técnica $\mathrm{CPC}$ con línea de terminación chamfer y bisel según tipo de corte y punto de medición marginal. En la terminación bisel tanto en el corte vestíbulo-palatino (A1:27,17 $\pm 21,11 \mu \mathrm{m}, \mathrm{F} 1: 48,66 \pm 37,58 \mu \mathrm{m}$ ), como en el corte mesio-distal (A2:62,16 $\pm 18,38 \mu \mathrm{m}, \mathrm{F} 2: 47,91$ $\pm 16,77 \mu \mathrm{m}$ ) se obtuvieron valores menores respecto a los promedios con la línea de terminación chamfer, en el corte vestíbulo-palatino (A1:89,65 $\pm 58,39 \mu \mathrm{m}$, $\mathrm{F} 1: 92 \pm 88,40 \mu \mathrm{m})$ como en el corte mesio-distal (A2:91,72 $\pm 67,13 \mu \mathrm{m}, \mathrm{F} 2: 97,66 \pm 50,59 \mu \mathrm{m})$. En tabla 2 se muestran los resultados descriptivos de la discrepancia interna de cofias metálicas unitarias de aleación Co-Cr obtenidos con la técnica CPC sobre líneas de terminación chamfer y bisel, según tipo de corte y punto de medición interno. Para la línea de terminación bisel en el corte vestíbulo-palatino a nivel axial se obtuvo valores promedios en el punto B1 de $54,76 \pm 21,02 \mu \mathrm{m}$ y en el punto E1 de $48,11 \pm$ $13,98 \mu \mathrm{m}$, siendo estos valores similares a los obtenidos en el corte mesio-distal, en B2 de 53,51 \pm $24,95 \mu \mathrm{m}$ y en E2 de 61,24 $\pm 20,82 \mu \mathrm{m}$. En el corte vestíbulo-palatino a nivel oclusal se obtuvo valores promedios de discrepancia interna en el punto $\mathrm{C} 1$ de $125,40 \pm 41,84 \mu \mathrm{m}$ y en D1 de $157,08 \pm 45,04 \mu \mathrm{m}$; en el corte mesio-distal a nivel oclusal se obtuvo valores promedios similares en $\mathrm{C} 2$ de $141,98 \pm 37,31 \mu \mathrm{m}$ y en D2 de $138,58 \pm 39,86 \mu \mathrm{m}$. El menor valor de la discrepancia interna en ambos cortes mesio-distal y vestíbulo-palatino fue a nivel axial en comparación a la zona oclusal. Para la línea de terminación chamfer en el corte vestíbulo-palatino a nivel axial se obtuvieron valores promedios en el punto B1 de

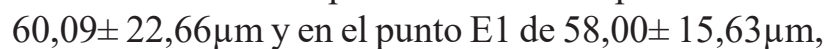
mientras que en el corte mesio-distal a nivel axial la discrepancia interna fue menor presentando valores en B2 de 42,30 $\pm 23,32 \mu \mathrm{m}$ y en E2 de 52,62 $\pm 26,16$ $\mu \mathrm{m}$. En el corte vestíbulo-palatino a nivel oclusal se obtuvo menores promedios de discrepancias internas en el punto $\mathrm{C} 1$ de $162,67 \pm 53,38 \mu \mathrm{m}$ y en $\mathrm{D} 1 \mathrm{de}$ $195,92 \pm 69,47 \mu \mathrm{m}$; en comparación con los valores encontrados en el corte mesio-distal en el punto $\mathrm{C} 2 \mathrm{de}$ $209,19 \pm 55,71 \mu \mathrm{m}$ y en D2 de 203,78 $\pm 62,94 \mu \mathrm{m}$. La menor discrepancia interna en ambos cortes mesiodistal y vestíbulo-palatino fue a nivel axial en 
Tabla1. Discrepancia marginal en cofias metálicas unitarias de aleación Co-Cr confeccionadas por la técnica de la cera perdida colado por centrifugación convencional sobre líneas de terminación chamfer y bisel según el tipo de corte y punto de medición marginal.

\begin{tabular}{ccccccccc}
\hline $\begin{array}{l}\text { Línea de } \\
\text { terminación }\end{array}$ & $\begin{array}{c}\text { Tipo de } \\
\text { corte }\end{array}$ & $\begin{array}{c}\text { Punto } \\
\text { Marginal }\end{array}$ & Min & Max & Promedio & D.E. & Mediana & DIC \\
\hline \multirow{2}{*}{ Bisel } & Vestibulo & A1 & 8,5 & 81,21 & 27,17 & 21,11 & 21,02 & 7,02 \\
& Palatino & F1 & 10,77 & 157,16 & 48,66 & 37,58 & 40,9 & 12,93 \\
& Mesio Distal & A2 & 32,05 & 92,29 & 62,16 & 18,38 & 65,87 & 14,01 \\
& & F2 & 12,95 & 79,56 & 47,91 & 16,77 & 49,48 & 7,26 \\
& Vestibulo & A1 & 32,77 & 219,94 & 89,65 & 58,39 & 57,93 & 76,08 \\
Chamfer & Palatino & F1 & 26,18 & 296,2 & 92,00 & 88,40 & 56,92 & 60,95 \\
& & A2 & 23,02 & 224,83 & 91,72 & 67,13 & 63,5 & 89,51 \\
& Mesio Distal & F2 & 38,62 & 202,63 & 97,66 & 50,59 & 84,42 & 64,78 \\
\hline
\end{tabular}

Prueba Shapiro Wilk

Min: mínimo.

Max: máximo.

DE: desviación estándar.

DIC: diferencia

intercuartiles

Tabla 2. Discrepancia interna en cofias metálicas unitarias de aleación Co-Cr confeccionadas por la técnica de la cera perdida colado por centrifugado convencional sobre líneas de terminación chamfer y bisel según tipo de corte y punto de medición interno

\begin{tabular}{cccccccccc}
\hline $\begin{array}{l}\text { Línea de } \\
\text { terminación }\end{array}$ & $\begin{array}{c}\text { Tipo de } \\
\text { corte }\end{array}$ & $\begin{array}{c}\text { Zona } \\
\text { interna }\end{array}$ & $\begin{array}{c}\text { Punto } \\
\text { Interno }\end{array}$ & Min & Max & Promedio & D.E. & Mediana & DIC \\
\hline \multirow{6}{*}{ Bisel } & \multirow{2}{*}{ Axial } & B1 & 34,31 & 104,64 & 54,76 & 21,02 & 45,79 & 25,88 \\
& Vestibulo & & E1 & 21,92 & 73,43 & 48,11 & 13,98 & 46,23 & 9,81 \\
& Palatino & \multirow{2}{*}{ Oclusal } & C1 & 43,71 & 190,25 & 125,40 & 41,84 & 122,41 & 43,73 \\
& & & D1 & 114,37 & 277,72 & 157,08 & 45,04 & 146,11 & 19,00 \\
& \multirow{2}{*}{ Axial } & B2 & 30,58 & 99,58 & 53,51 & 24,95 & 39,88 & 44,36 \\
& Mesio & & E2 & 16,94 & 94,20 & 61,24 & 20,82 & 64,97 & 26,00 \\
& Distal & \multirow{2}{*}{ Oclusal } & C2 & 95,33 & 203,31 & 141,98 & 37,31 & 139,71 & 52,04 \\
& & & D2 & 91,44 & 214,64 & 138,58 & 39,86 & 135,90 & 58,44 \\
& & \multirow{2}{*}{ Axial } & B1 & 29,32 & 100,43 & 60,09 & 22,66 & 54,61 & 14,43 \\
& & E1 & 35,42 & 79,57 & 58,00 & 15,63 & 61,03 & 21,24 \\
& Vestibulo & & C1 & 90,56 & 280,50 & 162,67 & 53,38 & 161,45 & 34,14 \\
& Palatino & \multirow{2}{*}{ Oclusal } & D1 & 115,30 & 331,73 & 195,92 & 69,47 & 166,99 & 73,99 \\
& & & B2 & 11,43 & 80,17 & 42,30 & 23,32 & 35,76 & 27,92 \\
& & \multirow{2}{*}{ Axial } & E2 & 24,26 & 114,87 & 52,62 & 26,16 & 44,45 & 22,71 \\
& Mesio & & C2 & 119,41 & 296,00 & 209,19 & 55,71 & 201,18 & 88,94 \\
& Distal & \multirow{2}{*}{ Oclusal } & D2 & 120,66 & 304,41 & 203,78 & 62,94 & 195,59 & 80,75 \\
\hline
\end{tabular}

Prueba de Shapiro Wilk

Min: mínimo.

Max: máximo.

DE: desviación estándar.

DIC: diferencia intercuartiles 
Tabla 3. Discrepancia marginal en cofias metálicas unitarias de aleación Co-Cr confeccionadas por la técnica de fresado de bloque blando en seco en CAD/CAM sobre líneas de terminación chamfer y bisel según tipo de corte y punto de medición marginal.

\begin{tabular}{lllllllll}
\hline $\begin{array}{l}\text { Línea de } \\
\text { terminación }\end{array}$ & $\begin{array}{l}\text { Tipo de } \\
\text { corte }\end{array}$ & $\begin{array}{l}\text { Punto } \\
\text { Marginal }\end{array}$ & Min & Max & Promedio & D.E. & Mediana & DIC \\
\hline \multirow{4}{*}{ Bisel } & Vestibulo & A1 & 20 & 62,92 & 45,18 & 13,65 & 46,65 & 7,88 \\
& Palatino & F1 & 28,28 & 61,1 & 44,52 & 10,13 & 45,91 & 6,98 \\
& Mesio & A2 & 25,12 & 87,13 & 35,34 & 16,29 & 29,69 & 3,15 \\
& Distal & F2 & 27,16 & 79,7 & 51,03 & 17,15 & 51,63 & 13,44 \\
& Vestibulo & A1 & 19,05 & 44,43 & 32,81 & 7,91 & 30,58 & 10,37 \\
\multirow{3}{*}{ Chamfer } & Palatino & F1 & 22,21 & 50,41 & 35,05 & 8,87 & 34,66 & 9,35 \\
& Mesio & A2 & 35,03 & 118,36 & 65,75 & 23,98 & 60,23 & 29,44 \\
& Distal & F2 & 40,5 & 115,6 & 70,18 & 24,40 & 66,59 & 31,94 \\
\hline
\end{tabular}

Prueba de Shapiro Wilk

Min: mínimo

Max: máximo.

DE: desviación estándar.

DIC: diferencia intercuartiles

Tabla 4. Discrepancia interna en cofias metálicas unitarias de aleación Co-Cr confeccionadas por la técnica de fresado de bloque blando en seco en CAD/CAM sobre líneas de terminación chamfer y bisel según tipo de corte y punto de medición interno.

\begin{tabular}{|c|c|c|c|c|c|c|c|c|c|}
\hline $\begin{array}{l}\text { Línea de } \\
\text { terminación }\end{array}$ & $\begin{array}{l}\text { Tipo de } \\
\text { corte }\end{array}$ & $\begin{array}{c}\text { Zona } \\
\text { interna }\end{array}$ & $\begin{array}{c}\text { Punto } \\
\text { Interno }\end{array}$ & Min & Max & Promedio & D.E. & Mediana & DIC \\
\hline \multirow{8}{*}{ Bisel } & \multirow{4}{*}{$\begin{array}{l}\text { Vestibulo } \\
\text { Palatino }\end{array}$} & \multirow{2}{*}{ Axial } & B1 & 33,42 & 104,25 & 70,71 & 24,52 & 75,20 & 19,34 \\
\hline & & & E1 & 38,28 & 120,77 & 73,06 & 24,57 & 70,60 & 20,04 \\
\hline & & \multirow{2}{*}{ Oclusal } & $\mathrm{C} 1$ & 48,66 & 218,56 & 108,98 & 48,56 & 101,05 & 24,56 \\
\hline & & & D1 & 40,49 & 216,75 & 105,58 & 46,71 & 109,89 & 30,75 \\
\hline & \multirow{4}{*}{$\begin{array}{l}\text { Mesio } \\
\text { Distal }\end{array}$} & \multirow{2}{*}{ Axial } & B2 & 35,28 & 153,31 & 66,94 & 32,74 & 57,80 & 15,47 \\
\hline & & & E2 & 53,75 & 155,95 & 110,91 & 32,66 & 113,88 & 20,92 \\
\hline & & \multirow{2}{*}{ Oclusal } & $\mathrm{C} 2$ & 35,93 & 280,93 & 107,32 & 59,39 & 95,61 & 22,29 \\
\hline & & & $\mathrm{D} 2$ & 37,60 & 237,78 & 97,60 & 47,40 & 88,23 & 14,34 \\
\hline \multirow{8}{*}{ Chamfer } & \multirow{4}{*}{$\begin{array}{l}\text { Vestibulo } \\
\text { Palatino }\end{array}$} & \multirow{2}{*}{ Axial } & B1 & 44,74 & 157,09 & 105,93 & 40,65 & 113,61 & 39,42 \\
\hline & & & E1 & 34,66 & 132,11 & 86,97 & 32,35 & 82,16 & 29,24 \\
\hline & & \multirow{2}{*}{ Oclusal } & $\mathrm{C} 1$ & 64,88 & 150,41 & 101,81 & 29,81 & 101,10 & 26,07 \\
\hline & & & D1 & 40,99 & 160,19 & 120,12 & 39,17 & 122,51 & 27,99 \\
\hline & \multirow{4}{*}{$\begin{array}{l}\text { Mesio } \\
\text { Distal }\end{array}$} & \multirow{2}{*}{ Axial } & B2 & 72,03 & 239,64 & 148,89 & 52,01 & 145,34 & 38,03 \\
\hline & & & E2 & 58,64 & 218,69 & 153,02 & 52,10 & 148,35 & 35,90 \\
\hline & & \multirow{2}{*}{ Oclusal } & $\mathrm{C} 2$ & 58,42 & 139,80 & 105,35 & 21,97 & 104,15 & 9,47 \\
\hline & & & D2 & 59,69 & 135,92 & 103,18 & 23,80 & 101,61 & 17,14 \\
\hline
\end{tabular}

Prueba de Shapiro Wilk

comparación a la zona oclusal. En la tabla 3 se muestran los promedios de la discrepancia marginal en cofias metálicas unitarias de aleación $\mathrm{Co}-\mathrm{Cr}$ realizadas por la técnica SMB con línea de terminación chamfer y bisel según tipo de corte y punto de medición marginal. En el corte vestíbulo-palatino, en la terminación bisel se obtuvo mayores valores en la discrepancia marginal en los puntos A1: 45,18 $\pm 13,65$ $\mu \mathrm{m}$ y F1: 44,52 $\pm 10,13 \mu \mathrm{m}$, en comparación con la

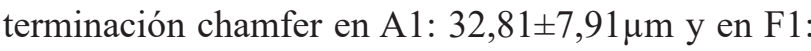


Tabla 5. Comparación de la adaptación marginal en cofias metálicas unitarias de aleación Co-Cr confeccionadas por la técnica de la cera perdida colado por centrifugación convencional y por fresado de bloque blando en seco en CAD/CAM con líneas de terminación chamfer y bisel, según tipo de corte y punto de medición marginal.

\begin{tabular}{|c|c|c|c|c|c|c|c|c|c|}
\hline \multirow[t]{2}{*}{$\begin{array}{c}\text { Línea de } \\
\text { terminación }\end{array}$} & \multirow[t]{2}{*}{$\begin{array}{l}\text { Tipo de } \\
\text { corte }\end{array}$} & \multirow[t]{2}{*}{$\begin{array}{c}\text { Punto } \\
\text { Marginal }\end{array}$} & \multicolumn{2}{|c|}{$\begin{array}{c}\text { Técnica de cera } \\
\text { pérdida colada } \\
\text { por centrifugación } \\
\text { convencional }\end{array}$} & \multicolumn{2}{|c|}{$\begin{array}{l}\text { Fresado de bloques } \\
\text { en seco CAD/CAM }\end{array}$} & \multirow[t]{2}{*}{$\begin{array}{l}\text { Diferencia } \\
\text { de medias }\end{array}$} & \multirow[t]{2}{*}{ Valor $\mathbf{p}$} & \\
\hline & & & Promedio & D.E. & Promedio & D.E. & & & \\
\hline \multirow{4}{*}{ Bisel } & Vestibulo & A1 & 27,17 & 21,11 & 45,18 & 13,65 & $-18,01$ & 0,0083 & ++ \\
\hline & Palatino & $\mathrm{F} 1$ & 48,66 & 37,58 & 44,52 & 10,13 & 4,13 & 0,6261 & ++ \\
\hline & Mesio & $\mathrm{A} 2$ & 62,16 & 18,38 & 35,34 & 16,29 & 26,82 & 0,0008 & ++ \\
\hline & Distal & $\mathrm{F} 2$ & 47,91 & 16,77 & 51,03 & 17,15 & $-3,12$ & 0,6432 & + \\
\hline \multirow{4}{*}{ Chamfer } & Vestibulo & A 1 & 89,65 & 58,39 & 32,81 & 7,91 & 56,84 & 0,0002 & ++ \\
\hline & Palatino & $\mathrm{F} 1$ & 92,00 & 88,40 & 35,05 & 8,87 & 56,95 & 0,0312 & ++ \\
\hline & Mesio & $\mathrm{A} 2$ & 91,72 & 67,13 & 65,75 & 23,98 & 25,97 & 0,6261 & ++ \\
\hline & Distal & $\mathrm{F} 2$ & 97,66 & 50,59 & 70,18 & 24,40 & 27,48 & 0,0905 & + \\
\hline
\end{tabular}

+Prueba t student

++ Prueba U Mann Whitney

significancia $\mathrm{p}<0,05$

$35,05 \pm 8,87 \mu \mathrm{m}$. En el corte mesio-distal en la terminación bisel se obtuvieron menores valores de discrepancias marginales en los puntos (A2: $35,34 \pm$ $16,29 \mu \mathrm{m}, \mathrm{F} 2: 51,03 \pm 17,15 \mu \mathrm{m})$ en comparación con los promedios obtenidos en línea de terminación chamfer que fueron (A2: $65,75 \pm 23,98 \mu \mathrm{m}, \mathrm{F} 2$ : $70,18 \pm 24,40 \mu \mathrm{m})$. En la tabla 4 se observan los promedios de la discrepancia interna en cofias metálicas unitarias de aleación Co-Cr realizadas por la técnica SMB con línea de terminación chamfer y bisel, según tipo de corte y punto de medición interno. Para la línea de terminación bisel los valores obtenidos en el corte vestíbulo-palatino a nivel axial fueron en el punto B1 de 70,71 $\pm 24,52 \mu \mathrm{m}$ y en el punto E1 de $73,06 \pm 24,57 \mu \mathrm{m}$, mientras que en el corte mesiodistal a nivel axial la discrepancia interna obtuvo valores promedios similares B2: $66,94 \pm 32,74 \mu \mathrm{m}$ y en E2 de 110,91 $\pm 32,66 \mu \mathrm{m}$. En el corte vestíbulopalatino a nivel oclusal se obtuvo valores promedios de discrepancia interna en el punto $\mathrm{C} 1$ de $108,98 \pm$ $48,56 \mu \mathrm{m}$ y en D1 de $105,58 \pm 46,71 \mu \mathrm{m}$; estos valores promedios fueron similares en el corte mesio-distal a nivel oclusal en $\mathrm{C} 2$ de 107,32 $\pm 59,39 \mu \mathrm{m}$ y en D2 de $97,60 \pm 47,40 \mu \mathrm{m}$. La menor discrepancia interna en ambos cortes vestíbulo-palatino y mesio-distal fue a nivel axial en comparación a la zona oclusal. Para la línea de terminación chamfer en el corte vestíbulopalatino a nivel axial se obtuvo valores promedios en el punto $\mathrm{B} 1: 105,93 \pm 40,65 \mu \mathrm{m}$ y en el punto E1 de
$86,97 \pm 32,35 \mu \mathrm{m}$, mientras que en el corte mesiodistal a nivel axial la discrepancia interna obtuvo mayores valores promedios en B2 de 148,89 \pm $52,01 \mu \mathrm{m}$ y en E2 de $153,02 \pm 52,10 \mu \mathrm{m}$. En el corte vestíbulo-palatino a nivel oclusal se obtuvo valores promedios de discrepancia interna en el punto $\mathrm{C} 1$ de $101,81 \pm 29,81 \mu \mathrm{m}$ y en D1 de $120,12 \pm 39,17 \mu \mathrm{m}$; en el corte mesio-distal a nivel oclusal se obtuvo valores promedios similares en $\mathrm{C} 2$ de 105,35 $\pm 21,97 \mu \mathrm{m}$ y en D2 de $103,18 \pm 23,80 \mu \mathrm{m}$. En la tabla 5 se comparó la adaptación marginal de las cofias metálicas unitarias de la técnica CPC y la técnica SMB para las líneas de terminación chamfer y bisel según tipo de corte y punto de medición marginal entre las dos técnicas. En la terminación bisel, en el corte vestíbulo-palatino se obtuvo valores en el punto A1 de $(p=0,0083)$ y F1 de $(p=0,6261)$, en el corte mesio-distal en los puntos A2 $\mathrm{de}(\mathrm{p}=0,0008)$ y $\mathrm{F} 2 \mathrm{de}(\mathrm{p}=0,6432)$. Para la terminación bisel en el sentido vestíbulo palatino sólo se observó diferencias estadísticamente significativas $(\mathrm{p}<0,05)$ en el punto A1 presentando una mejor adaptación marginal para la técnica CPC y en el corte mesiodistal se observó diferencias estadísticamente significativas $(p<0,05)$ en el punto A2 presentando una mejor adaptación marginal para la técnica SMB. Por lo tanto, para la terminación bisel los resultados no fueron concluyentes para determinar cuál de las dos técnicas es mejor en adaptación marginal para esta línea de terminación. En la terminación chamfer, en 
Tabla 6. Comparación de la adaptación interna en cofias metálicas unitarias de aleación Co-Cr confeccionadas por la técnica de la cera perdida colado por centrifugación convencional y fresado de bloque blando en seco en CAD/CAM con línea de terminación chamfer y bisel, según tipo de corte y punto de medida interna.

\begin{tabular}{|c|c|c|c|c|c|c|c|c|c|c|}
\hline \multirow[t]{2}{*}{$\begin{array}{c}\text { Línea de } \\
\text { terminación }\end{array}$} & \multirow[t]{2}{*}{$\begin{array}{l}\text { Tipo de } \\
\text { corte }\end{array}$} & \multirow[t]{2}{*}{$\begin{array}{c}\text { Zona } \\
\text { interna }\end{array}$} & \multirow[t]{2}{*}{$\begin{array}{c}\text { Punto } \\
\text { Interno } \\
\text { Promedio }\end{array}$} & \multicolumn{2}{|c|}{$\begin{array}{l}\text { Técnica de cera } \\
\text { pérdida colada } \\
\text { por centrifugación } \\
\text { convencional }\end{array}$} & \multicolumn{2}{|c|}{$\begin{array}{l}\text { Fresado de bloques } \\
\text { en seco CAD/CAM }\end{array}$} & \multirow[t]{2}{*}{$\begin{array}{l}\text { Diferencia } \\
\text { de medias }\end{array}$} & \multirow[t]{2}{*}{ Valor $\mathbf{p}$} & \\
\hline & & & & Promedio & D.E. & Promedio & D.E. & & & \\
\hline \multirow{8}{*}{ Bisel } & \multirow{4}{*}{$\begin{array}{l}\text { Vestibulo } \\
\text { Palatino }\end{array}$} & \multirow[t]{2}{*}{ Axial } & B1 & 54,76 & 21,02 & 70,71 & 24,52 & $-15,95$ & 0,0858 & ++ \\
\hline & & & E1 & 48,11 & 13,98 & 73,06 & 24,57 & $-24,95$ & 0,004 & + \\
\hline & & \multirow{2}{*}{ Oclusal } & $\mathrm{C} 1$ & 125,40 & 41,84 & 108,98 & 48,56 & 16,41 & 0,365 & + \\
\hline & & & D1 & 157,08 & 45,04 & 105,58 & 46,71 & 51,50 & 0,0023 & ++ \\
\hline & \multirow{4}{*}{$\begin{array}{l}\text { Mesio } \\
\text { Distal }\end{array}$} & \multirow{2}{*}{ Axial } & B2 & 53,51 & 24,95 & 66,94 & 32,74 & $-13,43$ & 0,1178 & ++ \\
\hline & & & E2 & 61,24 & 20,82 & 110,91 & 32,66 & $-49,67$ & 0,0001 & + \\
\hline & & \multirow{2}{*}{ Oclusal } & $\mathrm{C} 2$ & 141,98 & 37,31 & 107,32 & 59,39 & 34,66 & 0,0096 & ++ \\
\hline & & & D2 & 138,58 & 39,86 & 97,60 & 47,40 & 40,98 & 0,0044 & + \\
\hline \multirow{8}{*}{ Chamfer } & \multirow{4}{*}{$\begin{array}{l}\text { Vestibulo } \\
\text { Palatino }\end{array}$} & \multirow{2}{*}{ Axial } & B1 & 60,09 & 22,66 & 105,93 & 40,65 & $-45,84$ & 0,0016 & + \\
\hline & & & E1 & 58,00 & 15,63 & 86,97 & 32,35 & $-28,97$ & 0,0077 & + \\
\hline & & \multirow{2}{*}{ Oclusal } & $\mathrm{C} 1$ & 162,67 & 53,38 & 101,81 & 29,81 & 60,87 & 0,0015 & + \\
\hline & & & D1 & 195,92 & 69,47 & 120,12 & 39,17 & 75,79 & 0,0009 & ++ \\
\hline & \multirow{4}{*}{$\begin{array}{l}\text { Mesio } \\
\text { Distal }\end{array}$} & \multirow{2}{*}{ Axial } & B2 & 42,30 & 23,32 & 148,89 & 52,01 & $-106,58$ & $<0,0001$ & + \\
\hline & & & E2 & 52,62 & 26,16 & 153,02 & 52,10 & $-100,40$ & $<0,0001$ & + \\
\hline & & \multirow{2}{*}{ Oclusal } & $\mathrm{C} 2$ & 209,19 & 55,71 & 105,35 & 21,97 & 103,84 & $<0,0001$ & + \\
\hline & & & D2 & 203,78 & 62,94 & 103,18 & 23,80 & 100,60 & $<0,0001$ & + \\
\hline
\end{tabular}

+Prueba t student

++ Prueba U Mann Whitney

significancia $\mathrm{p}<0,05$

el corte vestíbulo-palatino se obtuvo valores en el punto A1 de $(p=0,0002)$ y F1 de $(p=0,0312)$, en el corte mesio-distal en los puntos A2 de $(p=0,6261)$ y F2 de $(p=0,0905)$. Para la terminación chamfer los valores numéricos de la adaptación marginal en sentido vestíbulo-palatino presentó diferencias estadísticamente significativas $(\mathrm{p}<0,05)$ en todos los puntos de medición en las cofias realizadas mediante SMB en comparación con la técnica CPC. En el corte mesio-distal los valores numéricos de la adaptación marginal en la línea de terminación chamfer fueron menores en la técnica SMB en comparación con la técnica CPC pero no había diferencias estadísticamente significativas, por lo tanto los resultados no fueron concluyentes para determinar cuál de las dos técnicas es mejor en adaptación marginal para esta línea de terminación. En la tabla 6 se comparó la adaptación interna de las cofias metálicas unitarias de la técnica CPC y la técnica SMB para las líneas de terminación chamfer y bisel según línea de terminación, tipo de corte, zona y punto de medición interno entre las dos técnicas. En la línea de terminación bisel, en el corte vestíbulo-palatino en la zona axial se obtuvo valores en el punto B1 de $(p=0,0858)$ y E1 de $(p=0,004)$, en la zona oclusal se obtuvo valores en el punto $\mathrm{C} 1 \mathrm{de}$ $(\mathrm{p}=0,365)$ y $\mathrm{D} 1 \mathrm{de}(\mathrm{p}=0,0023)$. En el corte mesiodistal en la zona axial se obtuvo valores en los puntos B2 de $(p=0,1178)$ y E2 de $(p=0,0001)$ en la zona oclusal se obtuvo valores en el punto $\mathrm{C} 2$ de $(\mathrm{p}=$ $0,0096)$ y D2 de $(p=0,0044)$. Los resultados mostraron diferencias estadísticamente significativas $(p<0,05)$ en los puntos E1, D1, E2, C2 y D2. Concluyendo que en esta línea determinación para ambos cortes los valores de la adaptación interna fue mejor a nivel axial para la técnica CPC, pero a nivel oclusal fue mejor la técnica SMB, pero sin diferencias estadísticamente significativas para ambas técnicas. En la línea de terminación chamfer, en el corte vestíbulo-palatino en la zona axial se obtuvo valores en el punto B1 de $(p=0,0016)$ y E1 de $(p=0,0077)$, en la zona oclusal se obtuvo valores en el punto $\mathrm{C} 1 \mathrm{de}$ $(p=0,0015)$ y $D 1 d e(p=0,0009)$. En el corte mesio- 
distal en la zona axial se obtuvo valores en los puntos B2 y E2 de $(p<0,0001)$, en la zona oclusal se obtuvo valores en el punto $\mathrm{C} 2$ y $\mathrm{D} 2$ de $(\mathrm{p}<0,0001)$. Los resultados mostraron diferencias estadísticamente significativas $(p<0,05)$ en todos los puntos, concluyendo que en esta línea de terminación para ambos cortes la adaptación interna fue mejor a nivel axial para la técnica CPC, pero a nivel oclusal fue mejor la técnica SMB. Los resultados obtenidos en la adaptación interna tanto en la zona axial como oclusal no fueron concluyentes para determinar que una técnica es mejor que la otra en adaptación interna en ambas líneas de terminación.

\section{DISCUSIÓN}

El presente estudio fue de tipo experimental, transversal y comparativo, tuvo el objetivo de comparar la adaptación marginal e interna de cofias metálicas unitarias de Co-Cr con línea de terminación tipo chamfer y bisel, confeccionadas por la técnica CPC y SMB.

Al comparar la adaptación marginal de las cofias metálicas unitarias $\mathrm{Co}-\mathrm{Cr}$ sobre las líneas de terminación chamfer y bisel entre la técnica SMB y $\mathrm{CPC}$, las que presentaron una mejor adaptación marginal para la terminación chamfer fue el SMB en comparación con la técnica $\mathrm{CPC}$ observándose diferencias estadísticamente significativas $(p<0,05)$ en sentido vestíbulo-palatino pero en sentido mesiodistal no hubo diferencias estadísticamente significativa, pero los valores promedios de la técnica SMB fueron menores, por lo tanto los resultados no fueron concluyentes para la línea de terminación chamfer. Para la adaptación marginal en la terminación bisel la técnica CPC en el sentido vestíbulo palatino sólo presentó diferencias estadísticamente significativas $(p<0,05)$ en el punto A1 comparada con el SMB y en el corte mesiodistal se observó diferencias estadísticamente significativas $(\mathrm{p}<0,05)$ en el punto A2 para la adaptación marginal en la técnica SMB comparándola con la técnica CPC. Por lo tanto, para la terminación bisel los resultados no fueron concluyentes para determinar cuál de las dos técnicas es mejor en adaptación marginal para esta línea de terminación.

La adaptación interna para la línea de terminación bisel en la técnica CPC, en la zona axial, mostraron diferencias estadísticamente significativas $(p<0,05)$ solo en los puntos E1 y E2 comparándola con la SMB. En la zona oclusal para la técnica SMB se obtuvo diferencias estadísticamente significativas $(p<0,05)$ solo en los puntos D1, C2 y D2 comparándola con la técnica CPC. La adaptación interna para la línea de terminación chamfer en los cortes vestíbulopalatino y mesio-distal en la zona axial mostraron diferencias estadísticamente significativas $(p<0,05)$ en todos los puntos de medición para la técnica CPC en comparación con la técnica SMB. En los cortes vestíbulo-palatino y mesio-distal en la zona oclusal mostraron diferencias estadísticamente significativas $(p<0,05)$ en todos los puntos de medición para la técnica SMB en comparación con la técnica CPC. Concluyendo que para ambas líneas determinación chamfer y bisel la adaptación interna fue mejor a nivel axial para la técnica CPC por tener menores valores numéricos que la técnica SMB pero sin diferencia estadísticamente significativa. Pero a nivel oclusal fue mejor la adaptación interna de la técnica SMB por tener menores valores numéricos que la técnica CPC pero sin diferencia estadísticamente significativa. Por lo tanto, los resultados obtenidos en la adaptación interna no fueron concluyentes para determinar que una técnica es mejor que la otra en adaptación interna. Con respecto a los métodos usados por los investigadores para evaluar la adaptación marginal e interna, la presente investigación coincide con muchos estudios como los de Nesse et al., (16), Xu et al., (12), y Huang et al., (2), en la utilización del método de réplica de silicona ya que proporcionó una medida fiable de la adaptación marginal por ser una técnica no destructiva permitiendo mediciones repetidas en múltiples puntos.

Bottino et al., en el 2007 realizaron un estudio donde evaluaron la adaptación marginal de cofias metálicas en acero inoxidable fabricadas para tres diferentes líneas de terminación: chamfer, hombro biselado a $135^{\circ}$ y hombro redondeado; fabricaron 180 cofias, 60 para cada grupo, luego cada grupo se dividió en tres subgrupos de 20 cada uno, usando tres diferentes agentes cementantes fosfato de zinc, ionómero de vidrio y cemento resinoso (4). Los resultados fueron que la línea de terminación chamfer obtuvo la mejor adaptación marginal independiente del tipo de cemento $(36,6 \pm 3,1$ a $100,8 \pm 4 \mu \mathrm{m})$; su conclusión fue que la mejor adaptación marginal se logró con la línea de terminación chamfer. Los resultados de nuestro estudio coincidieron con estas conclusiones 
porque la mejor adaptación marginal se logró con la técnica SMB con línea de terminación chamfer con promedios de $32,81 \pm 7,91 \mu \mathrm{m}$ a $35,05 \pm 8,87 \mu \mathrm{m}$, otra diferencia es que en nuestro estudio no se realizó ningún tipo de cementación.

Syu et al., (10), en 1993 realizaron un estudio el cual tuvo por objetivo evaluar si las diferentes líneas de terminación tenían alguna influencia en la adaptación marginal e interna (a $1 \mathrm{~mm}$ del margen) de cofias coladas, se tallaron tres incisivos centrales superiores con bisel $110^{\circ}$, hombro bisel de $45^{\circ}$ y chamfer, luego se confeccionaron mediante la técnica de colado convencional 30 cofias, 10 para cada grupo. Sus resultados fueron respecto a adaptación marginal: bisel de $110^{\circ}$ de $1,1 \pm 1,6 \mu \mathrm{m}$ (labial); $3,4 \pm 4,4 \mu \mathrm{m}$ (lingual). Hombro bisel de $45^{\circ}$ de 3,7 $\pm 4,1 \mu \mathrm{m}$ (labial); $0,6 \pm 0,9 \mu \mathrm{m}$ (lingual). Chamfer de $3,1 \pm 3,3 \mu \mathrm{m}$ (labial); $1,9 \pm 2,5 \mu \mathrm{m}$ (lingual). Respecto a adaptación interna (a $1 \mathrm{~mm}$ del margen): bisel de $110^{\circ}$ de 15,6 $\pm 16,1 \mu \mathrm{m}$ (labial más lingual); Hombro bisel a $45^{\circ}$ de $31,8 \pm 17,2 \mu \mathrm{m}$ (labial más lingual). Chamfer de $26,4 \pm 17,1 \mu \mathrm{m}$ (labial más lingual). Los resultados de nuestro estudio coinciden con respecto a la adaptación interna ya que no fueron concluyentes como para indicar que técnica logró la mejor adaptación interna y que el diseño de la línea de terminación no tuvo influencia en los valores de discrepancias internas.

Zelada comparó la adaptación marginal e interna de cofias metálicas unitarias de $\mathrm{Co}-\mathrm{Cr}$ sobre dos líneas de terminación tipo chamfer y bisel, fabricadas con las técnicas $\mathrm{SMB}$ y de fusión selectiva por láser en $\mathrm{CAD} /$ CAM(31). Los resultados que encontró fueron que las cofias metálicas de fusión selectiva por láser en $\mathrm{CAD} /$ CAM realizadas sobre la línea de terminación tipo chamfer obtuvieron mejor adaptación marginal que las cofias de la técnica SMB, respecto a la adaptación interna los resultados no fueron concluyentes como para indicar que línea de terminación presento mejor adaptación interna. Estos resultados coinciden con la presente investigación donde también se trabajó con el sistema $\mathrm{CAD} / \mathrm{CAM}$ y la técnica $\mathrm{CPC}$, obteniendo una mejor adaptación marginal con en el SMB en $\mathrm{CAD} / \mathrm{CAM}$ con la línea de terminación chamfer. Pero en la adaptación interna no fueron concluyentes para determinar que una técnica es mejor que la otra comparando ambas líneas determinación chamfer y bisel.
Loarte comparó la adaptación marginal e interna de cofias metálicas confeccionadas mediante dos técnicas: CPC y SMB en línea de terminación chamfer (32). Los resultados que encontró fueron que las cofias metálicas unitarias de aleación $\mathrm{Co}-\mathrm{Cr}$ confeccionadas por SMB, según tipo de corte y punto, presentaron mejor adaptación marginal que las cofias confeccionadas por la técnica CPC y al comparar la adaptación interna, según tipo de corte, zona y punto interno, las cofias confeccionadas por la técnica CPC presentaron mejor adaptación interna frente a las cofias confeccionadas con el SMB por CAD/CAM.

Vojdani et al., elaboraron un estudio con el propósito de evaluar la adaptación de cofias de Co-Cr con línea de terminación de hombro de $90^{\circ}$, confeccionadas por dos sistemas de fresado por CAD/CAM: Coritec 450i (imes-icore $\mathrm{GmBh}$, Leibolzgraben, Germany) y Ceramill ${ }^{\circledR}$ (Amann Girrbach AG, Koblach, Alemania) utilizando bloque duro sinterizado Coritec $\mathrm{Co}-\mathrm{Cr}$ disc (imes-icore $\mathrm{GmBh}$, Leibolzgraben, Germany) y bloque blando presinterizado Ceramill ${ }^{\circledR}$ Sintron (Amann Girrbach AG, Koblach, Alemania) respectivamente, donde se obtuvo un promedio de adaptación marginal para fresado de bloques blandos de $195 \pm 1 \mu \mathrm{m}$ y de adaptación interna de $143 \pm 1 \mu \mathrm{m}$; en cambio para fresado de bloques duros se obtuvo un promedio de adaptación marginal de $104 \pm 3 \mu \mathrm{m}$ y de adaptación interna de $77 \pm 1 \mu \mathrm{m}$ el procedimiento utilizaron 10 cofias metálicas de aleación $\mathrm{Co}-\mathrm{Cr}$ por cada sistema, con un ángulo de convergencia de $10^{\circ}$, con una fuerza de $20 \mathrm{~N}$ en las impresiones para obtener las réplicas de silicona, donde realizaron secciones tanto en sentido mesio-distal y vestíbulopalatino (17). Para nuestro estudio las técnicas y procedimientos fueron similares ya que también se utilizó réplicas de silicona con cortes en sentido mesio-distal y vestíbulo-palatino para la medición de la discrepancia marginal e interna, una fuerza de $15 \mathrm{~N}$ para la técnica de réplica de silicona, se utilizó 13 cofias metálicas unitarias de aleación $\mathrm{Co}-\mathrm{Cr}$ por cada sistema y se trabajó con un modelo maestro con un ángulo de convergencia de $12^{\circ}$.

Park et al., reportaron un estudio donde se evaluó la adaptación marginal e interna de cofias elaboradas de Co-Cr con línea de terminación chamfer con 3 sistemas: sistema de colado convencional, sistema de fresado por CAD/CAM de bloque duro, sinterizado selectiva láser, obteniendo mejores resultados en las cofias elaboradas por sistema de colado 
convencional con una adaptación marginal e interna respectivamente de $34,02 \pm 8,86 \mu \mathrm{m} ; 49,27 \pm 9,64 \mu \mathrm{m}$ seguido del sistema de fresado de bloque duro de $54,46 \pm 24,99 \mu \mathrm{m} ; 49,27 \pm 21,43 \mu \mathrm{m}$ por último sinterizado selectivo láser con $69,02 \pm 20,41 \mu \mathrm{m}$; $100,66 \pm 20,46 \mu \mathrm{m}$; encontrándose diferencias estadísticamente significativas (33). Estos resultados son diferentes al presente estudio donde se encontró que el SMB presinterizado Co-Cr Ceramill ${ }^{\circledR}$ Sintron (Amann Girrbach AG, Koblach, Alemania) por CAD/ CAM tuvo una mejor adaptación marginal en la línea de terminación chamfer.

Mediante la técnica de CAD/CAM podemos realizar la confección de cofias de una manera rápida y de manera más simple en comparación con la técnica convencional, sin embargo, los sistemas CAD/CAM introducen algunos pasos adicionales en el proceso de fabricación de las cofias, los cuales pueden traer como consecuencias la falta de exactitud en la adaptación, pasos tales como el escaneado, el diseño del software, el material y procesos para el fresado. Durante el proceso del diseño y escaneado (CAD), la delimitación virtual depende de la resolución del sistema del escaneado utilizado, este es un factor importante ya que a menudo no se tiene en cuenta variaciones menores en la línea de terminación y tiende a mezclar las irregularidades y sombras, las cuales se reconocen como una línea lisa y continua, dando como resultado una malla continua maciza y lisa, lo cual puede conllevar a la falta de adaptación ya sea marginal o interna, esto también puede conducir a interferencias de contacto en los bordes incisales o oclusales lo cual puede ser perjudicial en la zona marginal $(21,29)$.

Otro desafío para los sistemas CAD/CAM está en su fase del fresado o maquinado (CAM), si el software es capaz de definir una ubicación exacta de la línea de acabado y medirla en micras, el CAM sólo es capaz de fresar hasta el tamaño de la fresa más pequeño, por lo tanto, el CAM es incapaz de replicar exactamente irregularidades inferiores a las dimensiones de las fresas de tallado, esta información se viene discutiendo hasta la actualidad (29).

Beuer et al., reportaron que los procesos de sinterización, los procedimientos de escaneado, la recolección de datos geométricos, los parámetros de cálculo para el fresado y el proceso de tallado son factores que afectan en la adecuada adaptación marginal de restauraciones (34). Estos pueden ser factores que pudieron intervenir en los resultados de nuestro estudio, donde se encontró una mejor adaptación interna en la zona axial en ambas líneas de terminación en las cofias elaboradas por la técnica CPC comparada con el SMB por CAD/CAM, con diferencias estadísticamente significativas. Sin embargo, si bien es cierto la técnica del colado de patrones de cera es una técnica ampliamente utilizado debido a que nos permite una mejor manipulación y la posibilidad de conformar los patrones de cera precisamente, la cual será posteriormente eliminado completamente del molde mediante calentamiento, puede presentar varias limitaciones en el proceso de fabricación tales como: la sensibilidad térmica, memoria de elasticidad y el alto coeficiente de expansión térmica donde el proceso más delicado es la confección y colado de los patrones de cera y esta amerita el más mínimo cuidado (31). Estos factores pudieron conllevar que en nuestro estudio se encontró una mejor adaptación interna en la zona oclusal en ambas líneas de terminación en las cofias elaboradas por la técnica $\mathrm{SMB}$ por $\mathrm{CAD} / \mathrm{CAM}$.

Algunos estudios reportaron medidas mayores en la adaptación marginal e interna en sistemas CAD/CAM en comparación con los sistemas convencionales; sin embargo, también existen estudios donde los sistemas $\mathrm{CAD} / \mathrm{CAM}$ presentan una mejor adaptación marginal frente al colado convencional (21).

Kim et al., publicaron un estudio donde el objetivo fue medir y analizar la adaptación de las prótesis dentales fijas realizadas por diferentes sistemas dentro de ellas el SMB por CAD/CAM Co-Cr Ceramill ${ }^{\circledR}$ Sintron (Amann Girrbach AG, Koblach, Alemania) y el CPC; evaluaron un total de 10 cofias por grupo con línea de terminación chamfer y ángulo de convergencia de 12 grados (15), grupos con similares técnicas y procedimiento que nuestro estudio. La adaptación obtenida por la técnica SMB fue mejor que el CPC; siendo los resultados de discrepancia marginal de $32,6 \pm 4,8 \mu \mathrm{m}$ para el sistema de fresado y $64,1 \pm$ $14,2 \mu \mathrm{m}$ para el sistema convencional. En nuestro estudio también llegamos a la conclusión que la adaptación marginal de las cofias elaboradas por $\mathrm{SMB}$ por $\mathrm{CAD} / \mathrm{CAM}$ Co-Cr Ceramill ${ }^{\circ}$ Sintron (Amann Girrbach AG, Koblach, Alemania) presentan mejores resultados que las cofias elaboradas por CPC 
en la terminación chamfer.

La CPC es una técnica ampliamente utilizado debido a que nos permite una mejor manipulación, la posibilidad de conformar los patrones de cera precisamente, la cual será posteriormente eliminado completamente del molde mediante calentamiento, sin embargo este proceso de fabricación presenta varias limitaciones a saber tales como la sensibilidad térmica, memoria de elasticidad y el alto coeficiente de expansión térmica donde el proceso más delicado es la confección y colado de los patrones de cera y esta amerita el más mínimo cuidado.

Se reportó en estudios que el acto de remover los patrones de cera del molde puede con llevar a una variación de la adaptación marginal generando una apertura alrededor de $35 \mu \mathrm{m}$ (2). Otro factor que pudo haber intervenido en los resultados es el cambio dimensional de la impresión PVS que es de $0,05 \%$ y la expansión del yeso tipo IV usado en la confección de los modelos de trabajo que es de 0,05 a $0,07 \%$ (35). Una limitación del estudio fue no haber usado un modelo maestro preparado digitalmente y no hubo conflicto de interés. Se recomienda realizar investigaciones que permitan evaluar la adaptación marginal e interna mediante otras técnicas de fabricación, después del proceso de aplicación de la porcelana, con diferente línea de terminación, con diferente ángulo de convergencia, con otros materiales de fabricación de cofias, etc.

\section{CONCLUSIONES}

Según los resultados obtenidos la adaptación marginal e interna de cofias metálicas unitarias de aleación Co-Cr confeccionada mediante dos técnicas: CPC y $\mathrm{SMB}$, para la línea de terminación chamfer y bisel los resultados no fueron concluyentes para determinar cuál de las dos técnicas es mejor en adaptación marginal para ambas líneas de terminación. Ambas técnicas para la elaboración de cofias metálicas resultan ser eficientes en la adaptación marginal, los valores alcanzados se encontraron por debajo del valor clínico aceptable de $120 \mu \mathrm{m}$.

\section{Correspondencia:}

Enrique Manuel Gutiérrez Chanjón

Correo Electrónico : emaguch@hotmail.com

\section{REFERENCIAS BIBLIOGRÁFICAS}

1. Quante K, Ludwig K, Kern M. Marginal and internal fit of metal-ceramic crowns fabricated with a new laser melting technology. Dent Mater. 2008; 24(10):1311-5.

2. Huang Z, Zhang L, Zhu J, Zhao Y, Zhang X. Clinical marginal and internal fit of crowns fabricated using difefrent $\mathrm{CAD} / \mathrm{CAM}$ technologies. J Prosthodont. 2015; 24(4):291-5.

3. Wu L, Zhu H, Gai X, Wang Y. Evaluation of the mechanical properties and porcelain bond strength of cobalt-chromium dental alloy fabricated by selective laser melting. J Prosthet Dent. 2014; 111(1):51-5.

4. Bottino MA, Valandro LF, Buso L, Ozcan M. The influence of cervical finish line, internal relief, and cement type on the cervical adaptation of metal crowns. Quintessence Int. 2007; 38(7):425- 32.

5. Harish V, Mohamed A, Jagadesan N, Mohamed, Siva S. Evaluation of Internal and Marginal Fit of Two Metal Ceramic System - In Vitro Study. J Clin Diagn Res. 2014; 8(12): ZC53-ZC56.

6. Neclar D, Atiyu N, Meral A. Evaluation of the marginal fit of full ceramic crowns by the microcomputed tomography (micro-CT) technique. Eur J Dent. 2014; 8(4): 437-444.

7. Rosenstiel S, Land M. Prótesis Fija: Procedimientos clínicos y de laboratorio. Barcelona: Salvat Editores; 2009.

8. Pegoraro LF. Prótesis Fija. Sao Paulo: Artes Médicas; 2001.

9. Shillimburg H. Fundamentos esenciales en prótesis fija. Barcelona: Quintessence; 2006.

10. Syu JZ, Byrne G, Laub LW, Land MF. Influence of finish-line geometry on the fit of crowns. Int J Prosthodont. 1993; 6(1):25-30.

11. Mezzomo E. Rehabilitación oral contemporanea. Bogota: Amolca; 2010.

12. Xu D, Xiang N, Wei B. The marginal fit of selective laser melting fabricated metal crowns: An in vitro study. J Prosthet Dent.2014; 112(6): 1437-40.

13. Huang Z, Zhang L, Zhu J, Zhao Y, Zhang X. Clinical marginal and internal fit of crowns fabricated using difefrent $\mathrm{CAD} / \mathrm{CAM}$ technologies. J Prosthodont. 2015; 24(4):291-5.

14. Ortorp A, Jonsonn D, Moushen A, Steyern P. The fit of cobalt-chromium three-unit fixed dental prostheses fabricated with four different techniques: A comparative in vitro study. Dent Mater. 2011; 27(4):356-63.

15. Kim K, Kim J. Three-dimensional evaluation of gaps associated with fixed dental prostheses fabricated with new technologies. J Prosthet Dent.2014; 112(6): 143261.

16. Nesse H, Mari D, Myhre M, Øilo M. Internal and marginal fit of cobalt-chromium fixed dental prostheses fabricated with 3 different techniques. J Prosthet Dent. 
2015; 114(5):686-92.

17. Vojdani M, Torabi K, Farjood E, Khaledi AAR. Comparison the marginal and internal fit of metal copings cast from wax patterns fabricated by $\mathrm{CAD} /$ CAM and conventional wax up techniques. J Dent (Shiraz). 2013; 14(3):118-29.

18. Howard W, Berzins D, Keith Moore K, Charlton D. Metal-ceramic alloys in dentistry: A Review. J Prosthodont. 2009; 18: 188-194.

19. Geis-Gerstorfer J, Schille C, Schweizer E, Noack F, Hoffmann R. Comparison of the biocompatibility and corrosion properties of a $\mathrm{CoCr}$ sinter alloy with a casting alloy. Dental dialogue. 2013; 14: 20-5.

20. Li J, Ye X, Li B, Liao J, Zhuang P, Ye J. Effect of oxidation heat treatment on the bond strength between a ceramic and cast and milled cobalt-chromium alloys. Eur J Oral Sci. 2015; 123(4):297-304.

21. Lombardo G, Nishioka R, Souza R, et al. Influence of surface treatment on the shear bond strength of ceramics fused to cobalt-chromium. J Prosthodon. 2010; 19(2):103-11.

22. Hedberg Y, Wallinder I. Metal release and speciation of released chromium from a biomedical CoCrMo alloy into simulated physiologically relevant solutions. J Biomed Mater Res B Appl Biomater. 2014; 102(9): 693-699.

23. Johnson R, Verrett R, Haney S, Mansueto M, Challa S. Marginal gap of milled versus cast gold restorations. J Prosthodont. 2017; 26(1):56-63.

24. Abad J, Castro M, Durand E. Comparación de la adaptación marginal de cofias elaboradas en cuatro tipos de máquinas de colado. Revista Odontos. 2014; 43(4): 7-14.

25. Taggart W. A new Accurated method of making gold inlays. Dental Cosmos.1907; 49(11):1117-21.

26. Ammar A, Al S, Eanas I, Amer S. AI O. Marginal discrepancy of cobalt chromium metal copings fabricated with three different techniques. J Am Sci. 2015; 11(5):105-10.

27. Tamac E, Toksavul S, Toman M. Clinical marginal and internal adaptation of $\mathrm{CAD} / \mathrm{CAM}$ milling, laser sintering, and cast metal ceramic crowns. J Prosthet Dent. 2014; 112(4):909-13.

28. Wang H, Feng Q, Li N, Xu S. Evaluation of metalceramic bond characteristics of three dental $\mathrm{Co}-\mathrm{Cr}$ alloys prepared with different fabrication techniques. J
Prosthet Dent. 2016; 116(6): 916-13.

29. Ucar Y, Akova T, Akyil M, Brantley W. Internal fit evaluation of crowns prepared using a new dental crown fabrication technique: Laser - sintered Co-Cr crowns. J Prosthet Dent. 2009; 102(4):253-9.

30. Flores YE. Evaluación in vitro de las adaptaciones marginal e interna de cofias metálicas de aleación cobalto cromo $(\mathrm{Co}-\mathrm{Cr})$ sobre una línea de terminación tipo chamfer, confeccionadas con dos técnicas: cera pérdida colada por centrifugación convencional y el sistema fusión selectiva por láser asistido por computador (CAD/CAM).Tesis para obtener el título profesional de especialista en Rehabilitación Oral. Lima: Universidad Peruana Cayetano Heredia; 2017.

31. Zelada CC. Comparación in vitro de las adaptaciones marginal e interna en cofias metálicas unitarias de aleación cobalto cromo $(\mathrm{Co}-\mathrm{Cr})$ realizadas sobre dos líneas de terminación: tipo chámfer y tipo bisel, fabricadas con las técnicas de la cera perdida colado por centrifugado convencional y de fusión selectiva por láser, diseño asistido por computadora/manufactura asistida por computadora (CAD/CAM). Tesis para obtener el título profesional de especialista en Rehabilitación Oral. Lima: Universidad Peruana Cayetano Heredia; 2017.

32. Loarte MY. Comparación in vitro de la adaptación marginal e interna de cofias metálicas de aleación cobalto cromo (Co-Cr) confeccionadas con dos técnicas: de la cera perdida colada por centrifugación convencional y el sistema de fresado de bloques en seco asistido por computador (CAD/CAM). Tesis para obtener el título profesional de especialista en Rehabilitación Oral. Lima: Universidad Peruana Cayetano Heredia; 2017.

33. Park J, Wan W, Kim H, Kim J. Accuracy evaluation of metal copings fabricated by computer-aided milling and direct metal laser sintering systems. J Adv Prosthodont. 2015; 7(2):122-8.

34. Beuer F, Edelhoff D, Gernet W, Naumann M. Effect of preparation angles on the precision of zirconia crown copings fabricated by CAD/CAM system. Dent Mater J. 2008; 27(6):814-20.

35. Arpa M. Revisión de los últimos 10 años del ánalisis in vitro del ajuste marginal e interno de estructuras metálicas para prótesis fija dentosoportada. Tesis magister en Odontología. Madrid: Universidad Complutense de Madrid; 201

Recibido: 22-10-2018

Aceptado: 11-06-2019 